\title{
Necrotising Otitis Externa Antibiotic therapy complications: A retrospective cohort analysis
}

\author{
Ali Ijaz ${ }^{1}$, Emma Williams ${ }^{1}$, Joby Cole ${ }^{1}$, and Glen Watson ${ }^{1}$ \\ ${ }^{1}$ Sheffield Teaching Hospitals NHS Foundation Trust
}

December 27, 2021

\begin{abstract}
Introduction: Necrotising otitis externa (NOE) is a serious, progressive infection of the external ear canal. If untreated, it can invade into temporal bone, skull-base and surrounding tissue resulting in cranial nerve palsies, neurological infections and death. Patients present with unremitting, severe otalgia, otorrhoea and oedematous ear canals containing granulation. Surgery has a limited role; the mainstay of treatment involves a long course of intravenous antibiotics. Currently, there is no data on the complications of antibiotic treatment for NOE. This project aims to provide evidence on the nature/frequency of severe treatment-related complications requiring a change in antibiotic regime. Methods: A retrospective 5-year cohort analysis was performed on 64 patients who were treated for confirmed NOE with intravenous antibiotic therapy. Clinical notes, blood results and antibiotic prescriptions were documented and analysed. Results: Average duration of treatment was 11 weeks (range $=38$ weeks). There was an average of 2.1 antibiotic regimes per patient with 10 cases requiring inpatient admission due to treatment-related complications. $63 \%$ of treatment changes were directly related to adverse effects of intravenous antibiotics. Drug allergy/intolerance $(\mathrm{n}=18)$ and clinical deterioration i.e. Lack of symptomatic improvement and/or worsening inflammatory markers $(\mathrm{n}=18)$, were the most common reasons for antibiotic change. Neutropenia, deranged liver function tests and acute kidney injury were also recognised adverse effects of treatment. Conclusion: This study provides the first evidence on the notable frequency of antibiotic-related complications in NOE patients. Larger, multicentre studies are required in the future to validate our findings and will better inform both clinicians and patients of the risks of treatment.
\end{abstract}

\section{Abstract \\ Keywords: necrotising otitis externa, malignant otitis externa, antibiotic, complications}

Introduction - Necrotising otitis externa (NOE) is a serious, progressive infection of the external ear canal. If untreated, it can invade into temporal bone, skull-base and surrounding tissue resulting in cranial nerve palsies, neurological infections and death. Patients present with unremitting, severe otalgia, otorrhoea and oedematous ear canals containing granulation. Surgery has a limited role; the mainstay of treatment involves a long course of intravenous antibiotics. Currently, there is no data on the complications of antibiotic treatment for NOE. This project aims to provide evidence on the nature/frequency of severe treatmentrelated complications requiring a change in antibiotic regime.

Methods - A retrospective 5-year cohort analysis was performed on 64 patients who were treated for confirmed NOE with intravenous antibiotic therapy. Clinical notes, blood results and antibiotic prescriptions were documented and analysed.

Results - Average duration of treatment was 11 weeks (range $=38$ weeks). There was an average of 2.1 antibiotic regimes per patient with 10 cases requiring inpatient admission due to treatment-related complications. $63 \%$ of treatment changes were directly related to adverse effects of intravenous antibiotics. Drug allergy/intolerance $(\mathrm{n}=18)$ and clinical deterioration i.e. Lack of symptomatic improvement and/or wors- 
ening inflammatory markers $(\mathrm{n}=18)$, were the most common reasons for antibiotic change. Neutropenia, deranged liver function tests and acute kidney injury were also recognised adverse effects of treatment.

Conclusion - This study provides the first evidence on the notable frequency of antibiotic-related complications in NOE patients. Larger, multicentre studies are required in the future to validate our findings and will better inform both clinicians and patients of the risks of treatment.

\section{Key points}

- NOE is a rare but life-threatening condition. Treatment is long-term intravenous antibiotics. However, there is no evidence on the complications of antimicrobial treatment in this complex cohort of patients

- In our study, patients on average are initiated on two different antibiotic regimes

- $63 \%$ of these changes in regimen are due to direct adverse effects from treatment including drug intolerance and lack of significant clinical response leading to deterioration and morbid complications

- This novel data provides information clinicians can use when initiating treatment for NOE and counsel patients appropriately

- The increasing incidence of NOE with an ageing population and the rise in antibiotic resistances will likely further complicate management of this condition and prolong treatment duration

\section{Introduction}

Necrotising otitis externa (NOE), sometimes referred to as malignant otitis externa, is a rare but serious complication of acute otitis externa. It is a progressive infection of the external ear canal. If allowed to progress, there is a significant morbidity and mortality $(1)(2)(3)(4)$.

Patients usually present with severe unremitting otalgia, otorrhoea, hearing loss, and in more advanced cases cranial nerve palsy, neurological infection and sepsis. Well-established risk factors for NOE are age, diabetes and other conditions that compromise immune function. (1) First coined 'malignant' otitis externa in 1968 (5), incidence of the disease appears to be increasing. Pseudomonas aeruginosa has historically been the most common causative organism. More recently non-pseudomonal bacteria such as methicillin-resistant staphylococcus aureus (MRSA) and even fungal infections such as Candida are frequently being recognised $(6)(7)$.

The mainstay of treatment is long-term, high dose intravenous antibiotics (1)(8). Treatment duration may vary from 6 weeks to 3 months, dependent on individual patient risk factors (9). Although there is a role for local surgical debridement, the benefits are limited and it serves primarily to obtain deep samples for microbiology in resistant cases for those with severe complications or who are non-responsive to medical therapy $(10)(11)$.

Despite this prolonged treatment, there is no robust data on the complications of antibiotic-related therapy of NOE. Anecdotally, patients are required to change antimicrobial therapy multiple times throughout treatment as a direct result of these complications.

The objective of this study was to: Calculate the number of different antibiotic regimes typically used in the treatment of NOE and classify the different clinical reasons mandating a change in antibiotic therapy

\section{Methods}

\section{Ethical consideration and reporting}

A retrospective cohort analysis was performed on all patients with a diagnosis of necrotising otitis externa at "blinded for review" between June 2016 and January 2021. This data was collected and analysed in June 2021. STROBE guidelines were used for methodology. There are no ethical issues raised.

\section{Participants}

All patients with a confirmed diagnosis of necrotising otitis externa based on typical population risk groups, clinical findings, CT, and post contrast MRI scans were recorded. Patient characteristics such as age, gender 
and medical history were collected. Additionally, all patients baseline blood tests (full blood count, renal and liver function) prior to starting treatment were documented. Only patients requiring intravenous antibiotics as treatment were included in the analysis. All patients with NOE were discussed at MDT meetings involving ENT, microbiology and infectious disease teams to guide selection of antibiotic treatment.

\section{Data measurement}

Electronically scanned notes and prescriptions were used to record the antimicrobials prescribed for each patient. This included antibiotic prescribed and duration. All drug discontinuations and clinical rationale were documented. Any significant events over the duration of treatment such as hospital admissions or complications were also documented.

In our study, a single antibiotic regime was recorded as any prescribed antibiotics, given as either monotherapy or as combination antibiotic therapy. A change in regime was recorded when there was a switch in antibiotic prescription. This could be an addition, removal, or replacement of antibiotic.

All data was collected and analysed anonymously using Excel (Microsoft Corporation). Statistical analysis was performed using independent t-tests and Spearman's rank correlation with $95 \%$ confidence interval using SPSS v26 (IBM).

\section{Results}

\section{Participants:}

64 patients were identified on recruitment and one patient was excluded due to being managed on oral antibiotics. The cohort had a mean age of 74 (standard deviation (SD) \pm 13.7 ) years and a male: female ratio of 2.2:1. $70 \%$ of the group had a diagnosis of diabetes, of which $50 \%$ were treated with insulin. $11 \%$ of the cohort were at risk of immunosuppression due to long-term steroid use or regular immunosuppressant medication. eGFR was $62.3 \pm 19.4 \mathrm{ml} / \mathrm{min} / 1.73 \mathrm{~m}^{2}, 86 \%$ of patients had a chronic kidney disease staging of 2 to 3 (12). The mean Charlson Comorbidity Index score was 5 (SD \pm 1.99$)$. (Illustrated in table 1).

\section{Antibiotic Courses:}

There were a total number of 133 different intravenous antibiotic regimes given as treatment of NOE to this patient cohort. Mean number of intravenous antibiotic regimes per patient is $2.1 .41 \%$ received one antibiotic regime for the duration of treatment, $24 \%$ received two different antibiotic regimes, $22 \%$ received three different antibiotic regimes and $13 \%$ received four or five different antibiotic regimes.

A further 13 of the 63 patients (21\%) went on to complete the full treatment with oral antibiotics. The mean total days of treatment with intravenous antibiotics was 82.1 days $(\mathrm{SD} \pm 53.3$; median 67 days, range 266 days)

The mean duration of treatment in patients requiring one antibiotic regime, 61.1 days was significantly shorter $(\mathrm{p}=0.024)$ than those requiring two or more different antibiotic regimes, mean of 97.7 days.

When comparing risk factors; age was similar between the two groups $(\mathrm{p}=0.770)$. Diabetes $(\mathrm{p}<0.001)$ and insulin use $(\mathrm{p}<0.001)$ were significantly higher in patients requiring two or more different antibiotic regimes. There was no correlation or significant association between the Charlson Comorbidity Index and the number of courses required $(\mathrm{p}=0.093)$ or duration of treatment $(\mathrm{p}=0.158)$.

\section{Reasons for antibiotics changes:}

The reasons for changing antibiotic course are outlined in table 2. Adverse effects directly caused by intravenous antibiotic use represented over two-thirds $(68 \%)$ of the documented reasons mandating a change in medication The most common reasons were due to drug intolerance/allergy (19\%), clinical deterioration/rising inflammatory markers (19\%), microbiology advice and drug resistances (14\%) and deranged liver function tests (12\%). Further detail on microbiology advice is outlined in figure 3 . 
When accounting for multiple reasons for one change i.e., deranged liver function tests and thrombocytopenia, adverse effects from treatment represents $63 \%$ of all reasons for changing antibiotic therapy. There was a total of 10 inpatient admissions that were directly related to adverse effects of treatment.

Other non-injurious causes for antibiotic change were drug shortages, non-compliance, administrative error, and changes for outpatient antibiotic therapy (OPAT) suitability (18\% total).

\section{Antibiotic selection:}

A total of 9 different intravenous antibiotics were used in this patient cohort. The difference between monotherapy and combination therapy is illustrated in figure 4. Piperacillin with tazobactam (Tazocin) was the most used antibiotic, given as 69 separate courses (45\% of all intravenous antibiotic courses) and used as monotherapy in most cases (93\%). Ceftazidime (25\%), Teicoplanin (16\%) and Meropenem (9\%) were the next most common.

Oral and topical antibiotics commonly used were ciprofloxacin, amoxicillin and gentamicin.

\section{Discussion}

\section{NOE Cohort and antibiotic therapy}

Our results have shown that in our cohort of 63 patients, they have received, on average, two different intravenous antibiotic regimes (mean $=2.1$ courses). Over one third of patients $(35 \%)$ receive three or more intravenous courses. $63 \%$ of these changes are attributed to significant adverse effects from high-dose intravenous antibiotics. This does not include milder reactions or blood derangements that also occurred but did not necessitate a change in antibiotic. There were 10 hospital admissions directly related to adverse effects from treatment. 4 due to severe acute kidney infection (AKI), 2 due to deranged LFTs, 2 due to progression of NOE, and 1 for adverse reaction and clostridium difficile infection.

Recognised risk factors were prevalent in our cohort; with a mean age of 77 , predominantly male (44 male, 19 female). $70 \%$ had diabetes with half of this group taking insulin. These characteristics are similar to other population studies (1)(13)(14). A Charlson Comorbidity Index score of 5 is significantly higher than the average population (15) and reflects the general susceptibility this typical patient cohort has to developing NOE.

The intravenous antibiotics used most were tazocin $(45 \%)$ and ceftazidime $(25 \%)$. This is in line with other UK trusts; a survey on NOE antibiotic selection showed comparable figures, where Tazocin was used firstline in $41 \%$ of cases and ceftazidime in $7 \%$ (8). Similarly, treatment duration was 80.1 days on average, or a median of 65 days which better accounts for outliers. Although there is no agreed exact duration within the literature, this length of treatment appears consistent with other centres (9)(16). Therefore, both our cohort attributes and antimicrobial management appear representative of the UK NOE population.

We know that more medically complicated, multimorbid patients are likely to require longer treatment (13). 6 of the 8 patients requiring 4 or 5 intravenous antibiotic regimes were admitted as an inpatient and/or were treated with meropenem. The duration of treatment for those requiring multiple different antibiotic regimes (mean $=97.7$ days) was significantly longer $(p=0.024)$ than those treated successfully with one course $(61.1$ days). Diabetes was statistically more prevalent in those requiring multiple regimes. This may suggest that adverse effects of treatment may more frequently affect those with comorbidities and contribute to prolonged duration of treatment.

\section{The problems of treatment}

Antibiotic treatment is the globally accepted standard and delayed treatment has clearly identifiable complications (1)(17)(18). However, we are not aware of any current evidence on the complications of intravenous antibiotics in the NOE patient cohort.

It is well recognised that antibiotic use is associated with allergy, intolerance and adverse effects. Adverse events in short-term antibiotic use in hospitalised patients is as high as $20 \%$ with an increasing risk for every 
additional 10 days of use (19)(20). Prolonged use of tazocin is an independent risk factor for leukopenia and neutropenia; occurring in 10-16\% of cases (21). A large multi-centre study comparing tazocin and ceftazidime has shown that kidney injury, diarrhoea and rash occur with similar frequency (18-20\% risk cumulatively) (22).

However, this paper adds evidence specific to treatment within NOE patients. It shows that in complex patients with concomitant medical issues who will be on a prolonged course of treatment, these known adverse effects occur at a high rate. Uniquely, we have found the rate of alteration of treatment secondary to adverse effects to be remarkably high.

To our knowledge, we show the first study of antibiotic use in NOE, and we demonstrate that in these prolonged courses of antibiotics (mean $>60$ days) adverse effects are more common than in shorter courses of antibiotics. Intravenous antibiotic treatment (mean of 7 days) of 767 hospitalised patients with $P$. aeruginosa bacteraemia had a $1 \%$ discontinuation rate due to adverse effects (22). When including patients who did not respond to treatment in this group, this still only represents $38-46 \%$ vs $63 \%$ seen in our cohort. Long-term intravenous antibiotic use in other infective conditions found a $45 \%$ incidence rate of adverse drug effects and an increased duration of treatment in those affected (23). They did not report the frequency of antibiotic regime changes or dose alterations.

\section{Antibiotic resistances and microbiology}

Incidence of NOE is rising within the UK and with an increasing elderly population with multiple comorbidities, this trend is expected to continue (2)(6). There is also an increase in MRSA causing NOE with an associated decrease in Pseudomonas infection; which has previously been the most common causative organism (7)(13). This changing pathology mandates a change in antibiotic and may require longer treatment (7). The global rise in antibiotic resistance may further complicate treatment of this condition (24), $14 \%$ of our cohort required change in antibiotic therapy as a result of microbiological advice. This worrying pattern may mean more prolonged, multi-course antibiotic treatment in the future.

\section{Limitations}

Although this study provides novel data, it is primarily limited by cohort size. Larger groups would allow for risk stratification of patients to identify patient-specific risk factors that result in multiple courses of antibiotics or susceptibility to adverse treatment events. Additionally, multi-centre studies would reduce chance of error and offer statistical comparisons with our own cohort.

Adverse effects from treatment can also be caused by other confounding factors, particularly in this multimorbid patient group. Therefore, we did not count all abnormal results in our research to avoid this. We only evidenced drug discontinuations that clinicians felt was directly related to the antibiotic and subsequently required change. Additionally, other clinical deterioration and inpatient admissions that occurred during treatment that was not thought to be directly related to antibiotic treatment were excluded.

\section{Conclusion}

This retrospective cohort study adds new evidence to the significant and frequent complications of treatment in NOE. Identification of the commonly occurring issues and the potential impact this will have on antibiotic regimes, allows clinicians to counsel patients appropriately when initiating treatment. It can also increase awareness and inform appropriate monitoring for adverse events. Further data may help identify which patients may be more susceptible to these adverse effects and managed appropriately.

\section{Figure legend}

Figure/Table 1: Patient demographics and risk factors

Figure/Table 2: Reasons for changing antibiotic regime

Figure 3: Reasons for antibiotic change due to microbiology $(n=13)$ 
Figure 4: Antibiotic selection for treatment of NOE and use as mono or combined therapy

\section{References}

1. Treviño González JL, Reyes Suárez LL, Hernández de León JE. Malignant otitis externa: An updated review. Vol. 42, American Journal of Otolaryngology - Head and Neck Medicine and Surgery. Am J Otolaryngol; 2021.

2. Hopkins ME, Bennett A, Henderson N, Macsween KF, Baring D, Sutherland R. A retrospective review and multi-specialty, evidence-based guideline for the management of necrotising otitis externa. Vol. 134, Journal of Laryngology and Otology. J Laryngol Otol; 2020. p. 487-92.

3. Mahdyoun P, Pulcini C, Gahide I, Raffaelli C, Savoldelli C, Castillo L, et al. Necrotizing otitis externa: A systematic review. Vol. 34, Otology and Neurotology. Otol Neurotol; 2013. p. 620-9.

4. Frost J, Samson A. Standardized treatment protocol for necrotizing otitis externa: retrospective case series and systematic literature review. J Glob Antimicrob Resist. 2021 Jul 14

5. Chandler JR. Malignant external otitis. Vol. 78, Laryngoscope. Laryngoscope; 1968. p. 1257-94.

6. Chawdhary G, Liow N, Democratis J, Whiteside O. Necrotising (malignant) otitis externa in the UK: A growing problem. Review of five cases and analysis of national Hospital Episode Statistics trends. J Laryngol Otol. 2015 Jun 16;129(6):600-3.

7. Hobson CE, Moy JD, Byers KE, Raz Y, Hirsch BE, Mccall AA. Malignant Otitis Externa: Evolving Pathogens and Implications for Diagnosis and Treatment. Otolaryngol Neck Surg. 2014;151(1):112-6.

8. Pankhania M, Bashyam A, Judd O, Jassar P. Antibiotic prescribing trends in necrotising otitis externa: A survey of 85 trusts in the United Kingdom: Our Experience. Vol. 41, Clinical Otolaryngology. Clin Otolaryngol; 2016. p. 293-6.

9. Chawdhary G, Pankhania M, Douglas S, Bottrill I. Current management of necrotising otitis externa in the UK: survey of 221 UK otolaryngologists. Acta Otolaryngol. 2017 Aug;137(8):818-22.

10. Singh J, Bhardwaj B. The Role of Surgical Debridement in Cases of Refractory Malignant Otitis Externa. Indian J Otolaryngol Head Neck Surg. 2018 Dec 1;70(4):549-54.

11. Peled C, Parra A, El-saied S, Kraus M, Kaplan DM. Surgery for necrotizing otitis externa-indications and surgical findings. Eur Arch Oto-Rhino-Laryngology. 2020 Feb 12;277(5):1327-34.

12. Chen TK, Knicely DH, Grams ME. Chronic Kidney Disease Diagnosis and Management: A Review. Vol. 322, JAMA - Journal of the American Medical Association. NIH Public Access; 2019. p. 1294-304.

13. Arsovic N, Radivojevic N, Jesic S, Babac S, Cvorovic L, Dudvarski Z. Malignant Otitis Externa: Causes for Various Treatment Responses. J Int Adv Otol. 2020 Apr 1;16(1):98.

14. Sylvester MJ, Sanghvi S, Patel VM, Eloy JA, Ying YLM. Malignant otitis externa hospitalizations: Analysis of patient characteristics. Laryngoscope. 2017 Oct 1;127(10):2328-36.

15. Austin SR, Wong YN, Uzzo RG, Beck JR, Egleston BL. Why summary comorbidity measures such as the Charlson Comorbidity Index and Elixhauser score work. Med Care. 2015 ;53(9):e65-72.

16. Lullo AM Di, Russo C, Piroli P, Petti A, Capriglione P, Cantone E, et al. Malignant Otitis External: Our Experience and Literature Review. Am J Case Rep. 2020;21:e925060-1.

17. Hatch JL, Bauschard MJ, Nguyen SA, Lambert PR, Meyer TA, McRackan TR. Malignant Otitis Externa Outcomes: A Study of the University HealthSystem Consortium Database. Ann Otol Rhinol Laryngol. 2018 Aug 1;127(8):514-20.

18. Badaruddin A, Choo MM. Facial nerve palsy in otitis externa: A red flag? Malaysian Fam Physician Off J Acad Fam Physicians Malaysia. 2021 Feb 1;16(1):117. 
19. Tamma PD, Avdic E, Li DX, Dzintars K, Cosgrove SE. Association of adverse events with antibiotic use in hospitalized patients. JAMA Intern Med. 2017 Sep 1;177(9):1308-15.

20. Kokado R, Hagiya H, Koyama T, Matsui E, Okuno H, Morii D, et al. Antibiotic-associated adverse drug events at a Japanese academic hospital. J Infect Chemother. 2019 May;25(5):392-5.

21. Benli A, Şimşek-Yavuz S, Başaran S, Çağatay A, Özsüt H, Eraksoy H. Hematologic Adverse Effects of Prolonged Piperacillin-Tazobactam Use in Adults. Turkish J Hematol. 2018;35(4):290.

22. Babich T, Naucler P, Valik JK, Giske CG, Benito N, Cardona R, et al. Ceftazidime, carbapenems, or piperacillin-tazobactam as single definitive therapy for pseudomonas aeruginosa bloodstream infection: A multisite retrospective study. Clin Infect Dis. 2020 May 23;70(11):2270-80.

23. Kim DH, Kim HS, Nam KH, Choi BK, Han IH. Adverse Drug Reactions of Long-term Intravenous Antibiotics in Patients with Pyogenic Spondylitis. Korean J Spine. 2014;11(3):113.

24. Hand K. Antibiotic stewardship. Vol. 13, Clinical Medicine, Journal of the Royal College of Physicians of London. Royal College of Physicians; 2013. p. 499-503.

25. Marina S, Goutham MK, Rajeshwary A, Vadisha B, Devika T. A retrospective review of 14 cases of malignant otitis externa. J Otol. 2019 Jun;14(2):63. 
Cohort characteristic Patients $\mathrm{n}=63$

Mean age $( \pm S D) \quad 74( \pm 13.7)$ years

Male:Female ratio

44 Male: 19 Female

Diabetes (\%)

$44(70 \%)$

Immunosuppresed (\%)

$7(11.1 \%)$

Liver disease (\%)

$2(3.2 \%)$

eGFR ( \pm SD)

$62.3( \pm 19.4) \mathrm{ml} / \mathrm{min} / 1.73 \mathrm{~m}^{2}$

Mean Charlson Comorbidity Index ( \pm SD) 5 ( \pm 1.99 )

Table 1: Patient cohort demographics and risk factors 
Allergy/drug intolerance

Raised inflammatory markers/clinical deterioration

Micro advice/resistances

18.9

Deranged liver function test

11.6

OPAT suitability

11.6

Neutropenia

$8 \quad 8.4$

Acute kidney injury

Drug shortages

$\begin{array}{ll}7 & 7.4 \\ 3\end{array}$

Thrombocytopenia

Non-compliance

Administration error

Total reasons for antibiotic therapy change

Table 2: Reasons for changing antibiotic regime 
ANTIBIOTIC CHANGES DUE TO MICROBIOLOGY ( $N=13)$

- For further antimicrobial coverage $\quad$ Resistance identified

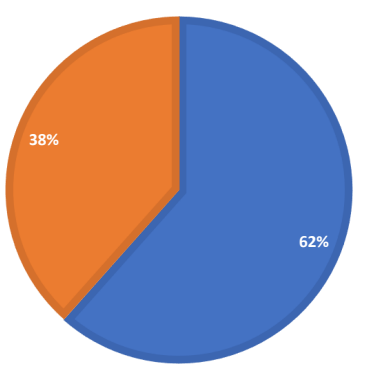

Figure 3: Reasons for antibiotic change due to microbiology 


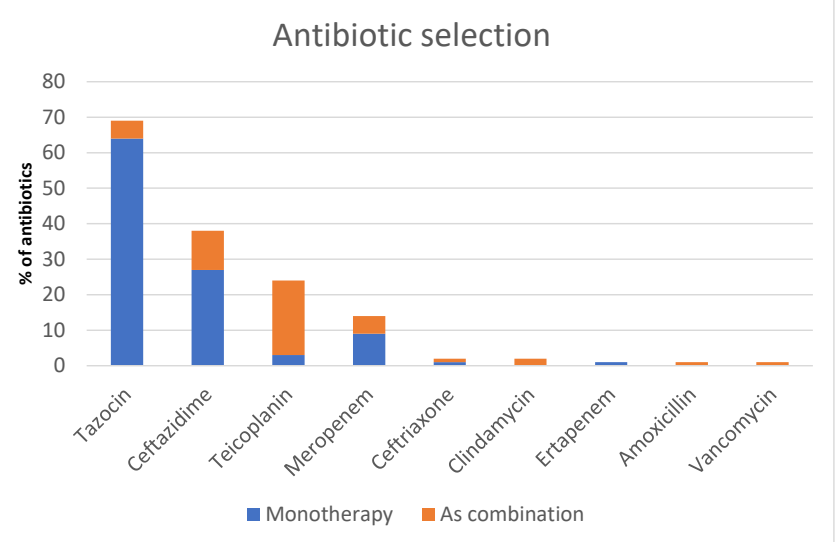

Figure 1: Antibiotic selection for treatment of NOE and use as mono or combined therapy 